\section{Summary of: Paediatric conscious sedation: views and experience of specialists in paediatric dentistry}

\author{
S. M. Woolley, ${ }^{1}$ E. J. Hingston, ${ }^{2}$ J. Shah ${ }^{3}$ and B. L. Chadwick ${ }^{4}$
}

\section{FULL PAPER DETAILS}

${ }^{1}$ Clinical Research Fellow in Restorative Dentistry, ${ }^{2} \mathrm{FTTA}$ in Paediatric Dentistry, ${ }^{3}$ General Dental Practitioner, ${ }^{4}$ Professor of Paediatric Dentistry, Applied Clinical Research and Public Health Group, Cardiff University School of Dentistry, Heath Park, Cardiff, CF14 4XY

${ }^{*}$ Correspondence to: Mr Stephen Woolley

Tel: +44 292074 4258; Fax: +44 2920743120

Email: woolleysm@cardiff.ac.uk

Online article number E11

Refereed Paper - accepted 19 December 2008

DOI: $10.1038 /$ sj.bdj.2009.664

${ }^{\circ}$ British Dental Journal 2009; 207: E11

\begin{abstract}
Objectives The objectives were three-fold: to investigate the level of conscious sedation training received prior to and during specialist training in paediatric dentistry; to establish the use of conscious sedation during and following specialisation; and to determine the attitudes of specialists in paediatric dentistry to conscious sedation. Subjects and methods A self-administered postal questionnaire was sent to all specialists in paediatric dentistry registered with the General Dental Council in January 2008. Non-responders were contacted again after a four-week period. Results A response rate of 60\% was achieved. Of the 122 respondents, 67 (55\%) had received sedation training as an undergraduate; 89 (75\%) had been trained during specialisation. All respondents performed dental treatment under sedation as a trainee and the majority used nitrous oxide inhalation sedation (NOIS). Over 90\% of respondents felt that NOIS should be available to all children, both in appropriate primary care settings and in hospitals. One hundred and twenty-one (99\%) respondents thought that all trainees in paediatric dentistry should have sedation training. Conclusions The most popular form of sedation amongst specialists in paediatric dentistry was NOIS. However, some of the respondents felt that children should have access to other forms of sedation in both the primary care and hospital settings. Additional research on other forms of sedation is required to evaluate their effectiveness and safety.
\end{abstract}

\section{EDITOR'S SUMMARY}

The issue of conscious sedation in dentistry, both for adults and for children, has seen considerable debate recently, as regular readers of the $B D J$ 'Letters' section will be aware. In any debate it is important to take into account the views of all parties concerned wherever possible, so in the area of paediatric conscious sedation it would seem logical to consider the views of specialists in paediatric dentistry (SPD), but until now their attitudes and experience have not been investigated, at least in the UK. The authors of this paper set out to remedy this omission.

The study looked at training in, use of and attitudes to conscious sedation among 122 SPD in the UK using a questionnaire. The majority of respondents had received sedation training during specialisation and half had received training as an undergraduate. All respondents had performed treatment on a patient under sedation while training, with most using nitrous oxide inhalation sedation (NOIS) - the most popular form of sedation among the specialists in the study, although some respondents also offered intravenous and oral sedation. The respondents' views on NOIS were interesting, with all of them stating that it should be available to children of all ages in a primary care setting when performed by dentists with appropriate training. The authors point out in their discussion that despite being noninvasive with few disadvantages, NOIS is not frequently used in general dental practice and suggest that it might be appropriate for non-specialists to provide NOIS, with specialists seeing the more challenging cases that may require more advanced techniques. However, the study found only limited support for advanced sedation techniques among paediatric specialists, despite their use by sedationists.

This paper makes a useful contribution to the continuing debate surrounding paediatric conscious sedation. Further research into other forms of sedation in paediatric dentistry is undoubtedly required, to evaluate their efficacy and safety and also to identify barriers to using NOIS alternatives among paediatric specialists. With dental anxiety so common among children, it is vital that the tools we have available to treat anxious patients are utilised in the most appropriate and effective way possible.

The full paper can be accessed from the $B D J$ website (www.bdj.co.uk), under 'Research' in the table of contents for Volume 207 issue 6.

Rowena Milan, Journal Editor DOI: 10.1038/sj.bdj.2009.835 


\section{TO ACCESS THE BDJ WEBSITE TO READ THE FULL PAPER:}

- BDA Members should go to www.bda.org.

- Click the 'login' button on the right-hand side and enter your BDA login details.

- Once you have logged in click the 'BDJ' tab to transfer to the BDJ website with full access.

IF YOUR LOGIN DETAILS DO NOT WORK:

- Get a password reminder: go to www.bda.org, click the login button on the right-hand side and then click the forgotten password link.

- Use a recommended browser: we recommend Microsoft Internet Explorer or Mozilla Firefox.

- Ensure that the security settings on your browser are set to recommended levels.

IF YOU HAVE NOT YET SIGNED UP TO USE THE BDA WEBSITE:

- Go to www.bda.org/getstarted for information on how to start using the BDA website.

\section{COMMENT}

When faced with the treatment of unco-operative patients such as very young infants, a teenager who is extremely afraid of injections, an individual with learning difficulties or others, the dentist is faced with the challenge of providing care that is safe and effective. If psychological techniques are inadequate, then conscious sedation or general anaesthesia may be considered.

The use of sedation is not standardised worldwide, and there are various modes of using sedation. This descriptive paper by Woolley et al. sheds some light on views and experience of specialists in paediatric dentistry in the UK with regards to paediatric conscious sedation. The information was obtained through a questionnaire which was sent to all specialists in paediatric dentistry (SPD) appearing on the General Dental Council register, followed by a second questionnaire to the non-respondents.

The authors provide a detailed description of the role of the respondents, the various treatments and the types of sedation provided by trainees and post-specialisation practitioners, the types of patients treated under sedation, the sedation methods in which SPD feel that trainees should be proficient, and the forms of sedation that SPD feel should be available to children in the primary care and hospital settings.

The authors rightfully raise the question as to when is the right time for training in sedation: during specialisation, prior to, or after acquiring some skills in paediatric dentistry? Not surprisingly, the most popular form of sedation amongst SPD was using nitrous oxide. However, some felt that other forms of sedation should be available to children in both primary care and hospital settings.

Although very detailed information is gathered in the study, some comparisons between groups would be interesting, and could have given more validity to the findings. Also, nearly one quarter of the references were national documents which are not available to all readers. Nevertheless, readers of this study are provided with an updated picture on the use of conscious sedation in the UK among SPD. This study is an important step towards standardisation of using conscious sedation in training, drugs used, monitoring, safety methods, and case selection.

\section{Professor B. Peretz}

Head, Department of Pediatric Dentistry, School of Dental Medicine,

Tel Aviv University, Israel

\section{AUTHOR QUESTIONS AND ANSWERS}

1. Why did you undertake this research? The initial idea for the research came from our awareness of the debate regarding appropriate sedation techniques in paediatric dentistry, especially with regard to the use of IV midazolam in children. A review of the literature revealed that the views of specialists in restorative dentistry regarding sedation training and use had been sought, but that the opinions of specialists in paediatric dentistry were unknown. We felt that this essential information, in addition to resources such as publications by governmental bodies and the sedation community would give a fuller picture of the clinical situation.

2. What would you like to do next in this area to follow on from this work?

This research gives an insight into sedation training and use prior to the publication of NICE guidelines. Despite the growing evidence for alternative techniques to inhalation sedation with nitrous oxide, this remains the preferred sedative modality for specialists in paediatric dentistry. Further research could assess the impact on practice of implementation of the guideline. Whilst at first glance, the non-invasive nature of nitrous oxide inhalation sedation may be thought to be the reason for this treatment trend, this should not be assumed to be the whole picture. Exploration of why specialists in paediatric dentistry prefer nitrous oxide, how they make decisions about its use and the impact of sedation modalities upon their practice lend themselves to future qualitative investigation. 